\title{
Krylov subspaces recycling based model order reduction for acoustic BEM systems and an error estimator
}

\author{
Dionysios Panagiotopoulos ${ }^{\mathrm{a}, \mathrm{b}, *}$, Elke Deckers ${ }^{\mathrm{a}, \mathrm{b}}$, Wim Desmet $^{\mathrm{a}, \mathrm{b}}$ \\ ${ }^{a}$ KU Leuven, Department of Mechanical Engineering, Celestijnenlaan 300 B, B-3001, \\ Heverlee, Belgium \\ ${ }^{b}$ DMMS lab, Flanders Make
}

\begin{abstract}
Boundary Element Method frequency sweep analyses in acoustics are usually accompanied by a vast numerical cost of assembling and solving numerous linear systems. In that context, this work proposes a model order reduction technique to mitigate the resulting computational cost of such analyses. First, a series expansion of the Green's function BEM kernel is leveraged to construct a series of frequency independent matrices. Next, in a model order reduction way, the arising matrices are projected on a reduced basis utilizing a Galerkin projection. By this off-line matrix projection, both the assembly and the solution of the BEM full-size linear systems degenerate into assembling and solving a reduced system for all frequencies. Significant speed-up factors can, thus, be achieved for both operations. The projection basis employed in this model reduction scheme is developed through an Arnoldi algorithm for the BEM systems on a grid of master frequencies. The method is based on Krylov subspaces recycling, as the subspaces produced at master frequencies are recycled to approximate the surface distribution of the acoustic variables on the whole frequency range of interest. Utilizing Krylov subspaces facilitates as well the definition of a robust error estimator that indicates the quality of the reduced system. The performance of the proposed method is assessed for both an exterior and an interior problem for a simple and more complicated geometry respectively.
\end{abstract}

\footnotetext{
${ }^{*}$ Corresponding author

Email address: dionysios.panagiotopoulos@kuleuven.be (Dionysios Panagiotopoulos)
}

Preprint submitted to Computer Methods in Applied Mechanics and EngineeringDecember 12, 2019 
Keywords: Model Order Reduction, BEM, Krylov subspaces recycling, Arnoldi, error estimator

\section{Introduction}

The Boundary Element Method 1, 2] (BEM) is an established numerical technique that is often used in the context of acoustic simulations. It is usually categorized by its formulation either as a direct or an indirect approach [3, 4].

5 The direct approach, on the one hand, describes the boundary conditions by physical quantities e.g. pressure, fluid normal velocity and its applicability is limited to closed geometries, with the exception of half-space problems with long geometries e.g. tunnel problems [5]. On the other hand, although the indirect formulation covers a broader range of applications by enabling modelling of open geometries for common problems as well, the boundary conditions are described by the pressure and normal velocity difference between its outer and inner face. Both approaches can be deployed either through a collocational scheme or by a Galerkin formulation [6, 7].

The BEM is regarded as an alternative technique to the Finite Element Method (FEM) in acoustic simulations. Comparing the two methods, the former relaxes the meshing requirement of a given $3 \mathrm{D}$ problem to a mere $2 \mathrm{D}$ mesh while the latter still demands a 3D mesh. Additionally, exploiting the BE formulation, the Sommerfeld radiation condition is automatically satisfied, while in the FE context it can only be fulfilled by employing techniques such as infinite elements [8] or the Perfectly Matched Layer (PML) [9, 10. Nevertheless, the FEM has proven to be more numerically efficient both in terms of required memory and of algorithmic efficiency to reach the solution of a time-harmonic acoustic analysis [11. In detail, although BEM benefits from a smaller number of Degrees of Freedom (DOFs) $N$, the produced matrices are not only fully populated and frequency dependent but also, in general, they do not demonstrate any regularity such as positive definiteness, symmetry, etc. [7. Thus, the computational demands for the storage of a BE system matrix amount to $\mathcal{O}\left(N^{2}\right)$, 
while the algorithmic complexity for the calculation of the solution scales between $\mathcal{O}\left(N^{2}\right)$ and $\mathcal{O}\left(N^{3}\right)$, depending on the employed linear systems solution algorithm (direct, iterative).

However, the computational cost grows considerably with the increase of frequency due to the meshing requirements. Specifically, as indicated by Marburg et al. [12, in certain applications the mesh refinement requirement can rise up to 10 elements per wavelength. In that context, several techniques to speed-up the BE solution have been developed. The most popular techniques are found in the combination of the Fast Multiple Method (FMM) [13, 14 and the $H$-matrices [15] with the BEM. The FMM-BEM method divides the geometry into a treestructure and by using a spherical wave expansion both the assembly and the solution of the BEM system are accelerated, while the $H$-matrix-BEM approach 40 is based upon the concept of hierarchical matrices and low rank approximations of the solution. Although these techniques relax the computational cost related to the storage and assembly of the BEM system to $\mathcal{O}(N \log N)$ and thus, perform well for large problems, they still require a system assembly and solution for each frequency line considered, which renders them highly time consuming for frequency sweep analyses.

In that way, additional techniques have been developed in an attempt to accelerate the fast frequency sweep analysis with the Boundary Elements as reported by Kirkup et al. [16]. These techniques simplify the BEM kernel aiming at avoiding to assemble the BEM system for each frequency line. In detail, discrete form interpolation strategies have come to the fore and are related to the Green's function frequency and space interpolation [17, 18, 19. Alongside, Taylor expansion of the Green's function has been exploited already since the early ' 80 [16, 20, 21], and has been further extended to account for the periodicity of the Green's function [22] and the indirect BEM formulation [23].

55 Despite the increasing popularity of these fast frequency sweep techniques based on interpolation strategies, they all demonstrate the same weaknesses. Specifically, as several master frequency systems or derivative matrices are necessary to approximate the system at intermediate frequencies, the memory re- 
quirements might rise to the extent of making the methods fail. Additionally, although the acceleration of the system assembly proves quite algorithmically efficient, despite the broadband character of the techniques, no specific strategy is employed to expedite the solution of the system. Hence, the offered acceleration by these techniques ends up being insignificant, especially in case of rather large BEM systems, where the solution of the system constitutes the most time-consuming part.

Aiming at alleviating these weaknesses, Lefteriu et al. 24 have combined the frequency interpolation technique by Schenck and Bethien [17, 18] with the Well-Conditioned Asymptotic Waveform Evaluation (WCAWE) introduced 25. and deployed by Slone in the framework of the finite element fast frequency 70 sweep analyses [26]. Specifically in [24, the frequency interpolation technique is leveraged not only to accelerate the assembly of the BEM system, but also to speed up the solution of the system through a Galerkin projection on momentmatching subspaces. Nevertheless, the disadvantage of this procedure lies on the fact that each system matrix needs to be projected in an online fashion 75 i.e. after its assembly. Thus, although the basis constructed by the momentmatching procedure is able to act universally inside each frequency window, an $N \times N$ system needs, first, to be assembled and then projected for each frequency. In addition to that, the recursive relation of the moments-vectors in combination with the computation of the derivative matrices "on-the-fly", so renders the creation of the projection basis costly.

In that context, this work introduces a novel Model Order Reduction (MOR) technique in conjunction with the Boundary Element analysis for acoustic problems. Although combining MOR with BEM can be regarded as an appealing idea, the development of such techniques is hindered due to certain bottlenecks. First, standard MOR-techniques are usually not applicable for BEM systems considering the heavily frequency dependent nature of BEM systems. In fact, constructing the representative basis might prove to be more cumbersome than the solution of the system itself. Krylov methods [27, 28, 29] that conveniently match the first moments of low order polynomial systems are not eligible in this 
case, as the complicated frequency dependency renders the moment matching a tedious procedure. Modal truncation methods that are often involved in vibroacoustics and dynamics model reduction [30, 31] cannot be employed in a straightforward manner as the cost of solving the non-linear eigenvalue problem formulated in Boundary Elements [32] would defeat the purpose of reducing the 95 model. Regarding the Proper Orthogonal Decomposition (POD) 33, 34, 35, although its flexibility and generality can make it applicable for BEM systems, the quality of the reduced model cannot be guaranteed, as it depends on the representativeness of the snapshots selected in the POD procedure [36].

Second, even obtaining a representative basis does not guarantee a significant reduction of the computational cost of a frequency sweep analysis. Namely, the projection of the assembled BEM system on the reduced basis in an online manner as conducted in [24, is cost-competitive to just solving the system by an iterative procedure such as GMRES [37. The latter is usually facilitated in BEM as it significantly speeds-up the solution procedure [38, 39. Some of the few attempts of joint BEM-MOR has been reported by Ryckelynck et al. 40] as an "a priori" reduction technique for potential problems in fluid mechanics. Finally, recently Liang et al. [41] proposed a MOR technique for coupled BEFE systems by constructing a modal projection basis through the Resolvent sampling based Rayleigh-Ritz method (RSRR) [42].

The idea of the method proposed in this work is based on finding a representative basis through an appropriately adjusted combination of POD and Krylov subspaces and subsequently, employing this basis for a Galerkin projection of the BEM system in an off-line fashion. In detail, an Arnoldi algorithm [43] is deployed for the BEM systems of a predefined master frequency grid to obtain Krylov subspaces of a certain order. These are exploited as the input snapshots for a POD algorithm which further selects the basis subspaces associated with the highest contribution. Hence, by expanding and accumulating these Krylov subspaces, a larger subspace that contains the subspace of approximated eigenvectors, i.e. the Ritz vectors [44 of the BEM system for each frequency, is created. The presented technique resembles to the Krylov subspaces recycling 
for varying systems [45] that was also recently employed in the context of a series of FMM-BEM acoustics systems [46]. Krylov subspaces recycling implies that the Krylov subspaces of the $j^{\text {th }}$ system are utilized for accelerating the convergence of the iterative solution procedure of the $(j+1)^{s t}$ system. In fact, ${ }_{125}$ Carlberg et al. in [47] and [48] have introduced a POD technique retaining all Krylov vectors produced for a series of related linear systems. Nonetheless, the yielded POD basis is only deployed to enrich the basis utilised in the iterative solution algorithm for each system independently and thus, accelerate the convergence of the iterative procedure and not as a global basis for the full set of linear systems in a model reduction scheme.

Analogous techniques that leverage a POD-Krylov combination have been reported in the literature. In certain works, Krylov subspaces up to third order are employed for the enrichment of the POD basis in a 2D fluid analysis [49, 40] and in 3D elasticity [50, 51]. Additionally, a Krylov-enhanced POD method (KPOD) [52] is also proposed where the moment-matching Krylov subspaces constitute the POD snapshots. Nevertheless, the basic motivation of these techniques is either the enrichment of the POD basis or the orthogonalization of the moment-matching subspaces respectively, while in the proposed technique the basic goal is to assemble a subspace containing the Ritz vectors 140 of neighbouring - in the frequency domain - BEM systems.

Subsequently to the construction of an appropriate basis, an off-line Galerkin projection is deployed on the BEM system. However, instead of projecting the newly assembled BEM systems, first, the Green's function Taylor expansion is leveraged as a frequency decoupling technique, as documented previously for MOR in conjunction with material frequency dependency in FEM [53]. Next, the resulting derivative matrices of the expansion are projected on the reduced basis. This is gradually performed for each power of the series expansion and at the end, a series of more compact matrices is obtained. Consequently, not only the algorithmic efficiency of the assembly and solution for each new BEM system ${ }_{150}$ is accelerated, but also the overall required memory scales more favourably as a function of the reduced model order $\ell$, tackling the previously reported memory 
problem arising from the combination of the BEM with Taylor Series [21]. This memory requirement relaxation can also prove advantageous in cases of large industrial models where even storing one full order BEM system is problematic.

Additionally, employing Krylov subspaces facilitates the derivation of an error estimator for the error induced due to the model reduction. Taking advantage of the fact that increasing the order of Krylov subspaces, the subspaces of a higher number of approximated system eigenvectors are assembled, the true relative error can be approximated by deploying a projection to a more detailed basis. The yielded distribution of the variables is compared to the one resulting from the more detailed reduction basis, instead of comparing to the solution of the full scale model. A similar procedure is followed in [54].

The method proposed in this work, constitutes a proof of concept for the combination of model order reduction with Boundary Elements. It selects a Taylor expansion for the frequency decoupling and a direct BEM formulation. There is no limitation in developing similar techniques leveraging different polynomial approximations of the kernel and concerning alternative BEM formulations such as the indirect BEM or the Burton-Miller approach [55]. However, depending on the recyclability of the generated Krylov subspaces, a different order of Krylov subspaces and density of master frequency grid might be required for the different BEM formulations, respectively.

The paper is organized as follows. In section 2, BEM is derived and the assembly of the system matrices through a collocational approach is demonstrated. Next in section 3 the Taylor expansion of the Boundary Element system and the off-line projection of the system onto a representative basis are discussed, along with a CHIEF method [56 projection framework. In section 4. the construction of a representative basis is elaborated and in section 5 an error estimator is introduced for the proposed MOR technique. In section 66 the method is first assessed with respect to the theoretical algorithmic complexities and then deployed on a simple benchmarking case and on a more industrially relevant model. Finally, section 7 summarizes and concludes the paper. 


\section{The direct Boundary Element Method with a collocational ap- proach}

In this section, the Boundary Element Method formulation utilised in this

185

$$
\begin{aligned}
c(\mathbf{y}) p(\mathbf{y}) & +\int_{\Gamma_{t o t}} \frac{\partial G(\mathbf{x}, \mathbf{y})}{\partial \mathbf{n}(\mathbf{x})} p(\mathbf{x}) d \Gamma_{t o t}(\mathbf{x})= \\
& =j \rho_{0} \omega \int_{\Gamma_{t o t}} G(\mathbf{x}, \mathbf{y}) u_{n}(\mathbf{x}) d \Gamma_{t o t}(\mathbf{x}), \quad \mathbf{y} \in \Omega_{t o t}, \mathbf{x} \in \Gamma_{t o t} .
\end{aligned}
$$

where $p(\mathbf{y})$ represents the pressure fluctuations induced by acoustic waves at any point $\mathbf{y}$ of the domain $\Omega_{t o t}, k=\frac{\omega}{c_{0}}$ is the acoustic wavenumber and $c_{0}$ the speed of sound in air.

Using the weak formulation of the above equation (1) and selecting the Green's function in $3 D$ for the test function, the well-known Boundary Integral equation can be derived as

Equation (2) is also known as the representation formula and it signifies that the pressure at any point $\mathbf{y}$ of the domain $\Omega_{t o t}$ can be calculated as a function of the acoustic variables on the boundary $\Gamma_{t o t}$. In equation (2) $u_{n}(\mathbf{x})$ and $\mathbf{n}(\mathbf{x})$ 


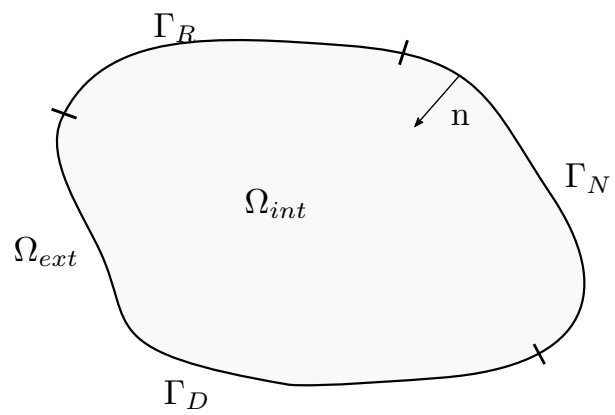

Figure 1: Domain definition for exterior problem

are the normal velocity of air and normal vector at point $\mathbf{x}$ of the geometry surface $\Gamma_{\text {tot }}$, respectively, and $r(\mathbf{x}, \mathbf{y})=|\mathbf{x}-\mathbf{y}| \cdot G(\mathbf{x}, \mathbf{y})$ is the Green's function in $3 \mathrm{D}$ and is defined as

$$
G(\mathbf{x}, \mathbf{y})=\frac{1}{4 \pi} \frac{e^{-j k r(\mathbf{x}, \mathbf{y})}}{r(\mathbf{x}, \mathbf{y})} .
$$

The function $c(\mathbf{y})$ indicates the participation factor and is calculated by the exterior solid angle at the position of $\mathbf{y}$; Referring at Figure 1, for points in the domain $\Omega_{e x t}$ it is unitary, in the domain $\Omega_{i n t}$ it is nullified, while for points on a smooth boundary $\Gamma_{t o t}$, it takes the value of $\frac{1}{2}$. In expression (2), the integrals containing the Green's function $G(\mathbf{x}, \mathbf{y})$ can be identified as the single layer potential, while the integrals containing the normal derivative of the Green's function $\frac{\partial G(\mathbf{x}, \mathbf{y})}{\partial \mathbf{n}(\mathbf{x})}$ constitute the double layer potential.

As illustrated in Figure 1, the boundary $\Gamma_{t o t}$ is divided into non-overlapping sections according to the boundary conditions characterizing it. In detail, $\Gamma_{D}$ signifies the boundaries with pre-described pressure, $\Gamma_{N}$ are boundaries with known normal velocity and $\Gamma_{R}$ represents boundaries with predefined normal impedance. Next, the boundary $\Gamma_{t o t}$ is discretized by a number of boundary elements $N_{e l}$ defined by the surface mesh nodes, as

$$
\Gamma_{t o t}=\bigcup_{i}^{N_{e l}} \Gamma_{i} .
$$

Having discretized the boundary, the acoustic variables are accurately repre- 
sented on the nodes and approximated by interpolation in between the nodes. In this work, piecewise linear polynomials are employed as interpolation functions $\Phi_{i}(\mathbf{x})$ to describe the acoustic variables as

$$
\begin{gathered}
p(\mathbf{x})=\sum_{i}^{N_{\phi}} \Phi_{i}(\mathbf{x}) p_{i}, \\
u_{n}(\mathbf{x})=\sum_{i}^{N_{\phi}} \Phi_{i}(\mathbf{x}) u_{n, i},
\end{gathered}
$$

225

\subsection{The collocation approach}

By employing the discretization strategy, the only unknowns of the problem are the nodal values of the acoustic variables. Thus, collocating the position of vector $\mathbf{y}$ to all nodes, the BEM system of equations is derived as

$$
\mathbf{H p}=\mathbf{G u}_{\mathbf{n}}
$$

230

where $\mathbf{G}, \mathbf{H} \in \mathbb{C}^{N \times N}$ represent the contribution of the whole boundary on the single and double layer potential respectively. Deploying the boundary conditions defined as per Figure 1 and rearranging, system (6) takes the form a linear system of equations given by

$$
\mathbf{A}(\omega) \boldsymbol{\xi}(\omega)=\mathbf{b}(\omega)
$$

where $\mathbf{A} \in \mathbb{C}^{N \times N}$ and $\boldsymbol{\xi}, \mathbf{b} \in \mathbb{C}^{N}$, which can be solved by a direct or iterative 235 solver. The yielded system (7) is frequency dependent and needs to be assembled and solved for each frequency line under consideration.

\section{Series Expansion BEM and Model Order Reduction}

Assembling systems like (7) is often quite computationally demanding. Hence, to alleviate the respective computational cost a series expansion technique can 
lems. In this section, a series expansion method for the direct collocational BEM is derived and the combination with a Galerkin projection is demonstrated. Finally, the procedure is adjusted to account as well for an appropriate treatment for the non-uniqueness problem through a valid CHIEF overdetermination tech245 nique 56 .

Series expansion of a BEM system might be conducted leveraging different polynomial expansion or interpolation techniques, such as Taylor expansion, Lagrange and Chebyshev polynomials [58. In this work, a Taylor expansion of the Green's function kernel is selected as it enables analytical evaluation of the polynomial coefficients. Nevertheless, as polynomial interpolation methods are based on the assembled systems at certain frequencies, they can be utilised to save on the additional assembly time needed for the derivative matrices of the Taylor expansion and to generate a less intrusive procedure.

\subsection{System assembly acceleration}

The basic idea of the Series Expansion BEM (SEBEM) relies on the Taylor expansion of the frequency-dependent part i.e. the BEM kernel. In detail, the coupled term $e^{-j k r}$ can be approximated through a Taylor expansion around a preselected wavenumber $k_{0}$ by

$$
e^{-j k r}=e^{-j k_{0} r} \sum_{m=0}^{M_{\max }} \frac{(-j r)^{m}}{m !}\left(k-k_{0}\right)^{m},
$$

where $M_{\max }$ represents the order of the series expansion.

Introducing the above expansion in the BEM integrals, the single and double layer potentials can be reformulated and substituted by a series of integrals depending only on $r$. The single layer potential approximation is given by

$$
\begin{aligned}
\int G(\mathbf{x}, \mathbf{y}) d r & =\frac{1}{4 \pi} \int \frac{e^{-j k r}}{r} d r= \\
& =\frac{1}{4 \pi} \sum_{m=0}^{M_{\max }} \frac{\left(k-k_{0}\right)^{m}}{m !} \int \frac{e^{-j k_{0} r}}{r}(-j r)^{m} d r
\end{aligned}
$$


while the double layer potential can be reconstructed analogously.

Transferring the series approximation of the Green's function to the system matrix level, implies the transformation of the system into a series of frequency decoupled matrices $\mathbf{G}_{m}, \mathbf{H}_{m} \in \mathbb{C}^{N \times N}$, each one corresponding to a different exponent of the frequency term $\left(k-k_{0}\right)^{m}$. Hence, the BEM system of equations (6) and (7) takes the form

$$
\begin{gathered}
\left(\sum_{m=0}^{M_{\max }} \frac{\left(k-k_{0}\right)^{m}}{m !} \mathbf{G}_{m}\right) \mathbf{u}_{\mathbf{n}}=\left(\sum_{m=0}^{M_{\max }} \frac{\left(k-k_{0}\right)^{m}}{m !} \mathbf{H}_{m}\right) \mathbf{p} \\
\left(\sum_{m=0}^{M_{\max }} \frac{\left(k-k_{0}\right)^{m}}{m !} \mathbf{A}_{m}\right) \boldsymbol{\xi}(\omega)=\sum_{m=0}^{M_{\max }} \frac{\left(k-k_{0}\right)^{m}}{m !} \mathbf{b}_{m}
\end{gathered}
$$

respectively.

Expressing the BEM system as a series of frequency decoupled matrices accelerates the assembly of the system at wavenumbers different than $k_{0}$. Nevertheless, the efficiency of this procedure is limited due to the complexity of assembling and storing all the derivative matrices of the system. In fact, this expansion starts being more cost efficient than regular BEM in cases that the number of derivative matrices $M_{\max }$ used in the series expansion is lower than the number of frequencies $N_{f}$ the system needs to be solved for, namely $M_{\max } \leq N_{f}$. In that sense, it constitutes a fast frequency sweep algorithm for BEM systems.

However, the excessive memory that is needed for this method constitutes the bottleneck in case of large systems. As all the $N \times N$ derivative matrices are needed simultaneously for the assembly of the BEM system at one frequency, the memory requirements easily exceed the available memory and thus, broad use of this technique is hindered.

In that context, this work proposes to combine the SEBEM approximation method with a suitable Model Order Reduction technique to scale down the 285 requirements of BEM both with respect to memory and algorithmic complexity. 


\subsection{Order of Series Expansion approximation}

The frequency range validity of SEBEM depends on the order of the Taylor expansion $M_{m a x}$, the selected expansion point $k_{0}$ and the maximum distance $r_{\max }$ occurring in the geometry. The residual at a specific wavenumber $k$ induced due to the Taylor approximation of the Green's function is given by [59]

$$
\begin{aligned}
\sigma(k) & =\left|e^{-j k_{n} r_{\max }} \frac{\left(-j r_{\max }\right)^{M_{\max }+1}}{\left(M_{\max }+1\right) !}\left(k-k_{0}\right)^{M_{\max }+1}\right| \\
& \leq\left|\frac{\left(-j r_{\max }\right)^{M_{\max }+1}}{\left(M_{\max }+1\right) !}\left(k-k_{0}\right)^{M_{\max }+1}\right|,
\end{aligned}
$$

where $k_{n}$ is a wavenumber between $k_{0}$ and $k$.

A low residual $\sigma$ at a specific frequency indicates that the SEBEM can still be considered valid. In Figure 2 the resulting maximum frequency interval $\Delta f_{\max }$ for a pre-defined residual upper bound of $\sigma=0.01$ is illustrated. The order $M_{\max }$ of respective Taylor expansion of the Green's function, as well as the potential maximum distance $r_{\max }$ between two nodes of the geometry constitute the contributing parameters. Hence, it is possible to consider in advance the desired frequency range of validity, which can be broadened by employing a multi-point Taylor expansion.

Deploying a multi-point Taylor expansion can also alleviates any potential concerns regarding the numerical stability of the high order polynomial that approximates the well-behaved, though highly oscillatory kernel. Although, for the parameter values of Figure 2 numerical stability of the polynomial expansion does not prove to be an issue, a combination of a longer maximum distance $r_{\max }$ with a high order expansion $M_{\max }$, might induce a considerable numerical error in the approximated kernel. In that case, a multi-point expansion can compress the expansion orders of all Taylor expansions, ensuring in parallel a stable behaviour. Finally, leveraging a multi-point Taylor expansion allows for the utilization of meshes of different refinement. Depending on the validity of 310 each mesh for different frequency spectra, a coarser mesh can be used for the lower frequency range, while a more refined one for the higher frequency range. 


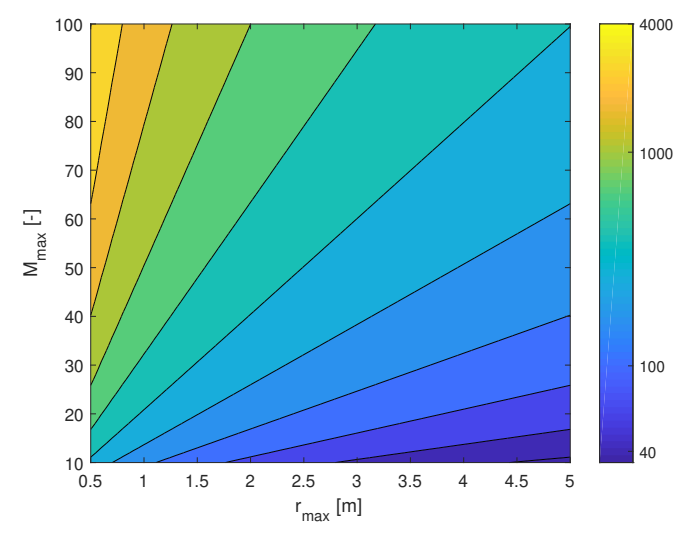

Figure 2: $\Delta f_{\max }[\mathrm{Hz}]$ for maximum residual $\sigma_{\max }=0.01$, considering $c_{0}=343 \frac{\mathrm{m}}{\mathrm{s}}$.

\subsection{Galerkin Projection of a SEBEM system}

Expanding the BEM system in a series does not offer a great advantage as it leads to excessive memory consumption. Nonetheless, combining a Galerkin projection technique with the SEBEM approach, substantially improves this method. Specifically, deploying a Galerkin projection to the derivative matrices $\mathbf{G}_{m}$ and $\mathbf{H}_{m}$ reduces the size of the system and as a result, cuts down drastically the resources required to store the sequence of original full size matrices.

The Galerkin projection is based on expressing the full system by its pro${ }_{320}$ jection on a lower dimensional subspace $\mathbf{V}_{\ell} \in \mathbb{C}^{N \times \ell}$, the assembly procedure of which is described in a section 4. In case of the BEM system of (6), the degrees of freedom $\boldsymbol{\xi}$ are approximated as

$$
\boldsymbol{\xi} \approx \hat{\xi}=\mathbf{V}_{\ell} \boldsymbol{\xi}_{\ell}
$$

where $\xi_{\ell}$ represents the degrees of freedom of the reduced system.

Introducing the approximation of (13) in (7) would yield an overdetermined system. By left-multiplying the yielded system with a left projection matrix $\mathbf{W}_{\ell} \in \mathbb{C}^{\ell \times N}$, the system matrix regains its square form, this time obtaining a 
much smaller dimension $\ell \ll N$ as illustrated by

$$
\mathbf{W}_{\ell} \mathbf{A V}_{\ell} \boldsymbol{\xi}_{\ell}=\mathbf{W}_{\ell} \mathbf{b},
$$

or equivalently by

$$
\mathbf{A}_{\ell}(\omega) \boldsymbol{\xi}_{\ell}(\omega)=\mathbf{b}_{\ell}(\omega),
$$

where $\mathbf{A}_{\ell} \in \mathbb{C}^{\ell \times \ell}$ and $\mathbf{b}_{\ell} \in \mathbb{C}^{\ell}$ are the system matrix and right hand-side of the

330 derivative matrix under assembly. However, as the reduced matrices can be

with $\mathbf{A}_{\ell m} \in \mathbb{C}^{\ell \times \ell}$ and $\mathbf{b}_{\ell m} \in \mathbb{C}^{\ell}$.

The polynomial system of (17) is now of lower dimension $\ell \ll N$ and thus, the system that needs to be assembled and solved at each frequency is more concise. In that sense, the above projection is conducted in an off-line manner, meaning that the system is reduced even before the assembly.

Additionally, the algorithmic implementation is of particular interest as it alleviates the previously reported issue of excessive memory consumption for the SEBEM. As illustrated in Algorithm (1), the derivative matrices $\mathbf{A}_{m}$ are directly projected onto the already produced Galerkin basis $\mathbf{V}_{\ell}$ after their assembly.

345 In that way, it is only necessary to allocate memory for the series of already reduced derivative matrices $\mathbf{A}_{\ell m}$, in addition to the memory needed for the 


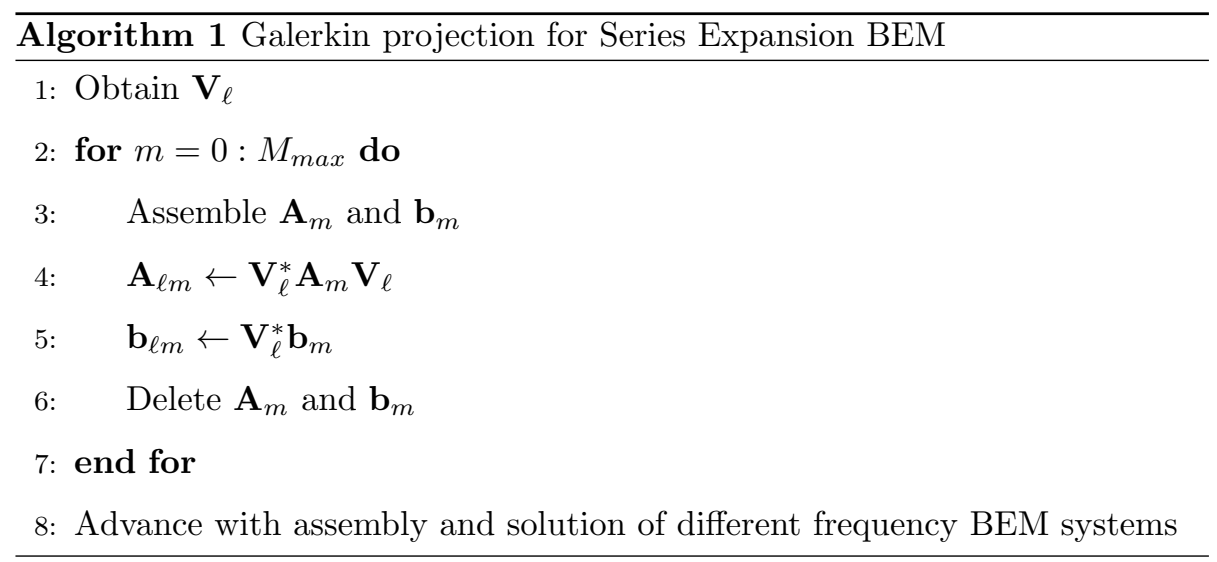

directly stored in the disk, the only memory required in the workspace during the construction of the reduced system is related to the storage of the projection matrices and, following a column by column projection, the one-side projection of the derivative matrix processed, namely $\mathcal{O}(2 N \times \ell)$ and $\mathcal{O}(N \times \ell)$ respectively. Upon projection of all derivative matrices, at the online stage of the assembly of the reduced system, the required storage scales with $\mathcal{O}\left(\ell^{2} \times M_{\max }\right)$, which is the memory required for storing the series of $\mathbf{A}_{\ell m}$ matrices.

355 3.4. The CHIEF in the model order reduction SEBEM

Considering exterior acoustic problems, the well documented non-uniqueness issue arises 60]. Specifically, for a set of so-called irregular frequencies the integral equation given in (2) becomes singular. Thus, the respective system of equations (7) does not yield a unique solution resulting in a set of fictitious resonances. Dealing with this issue, Schenck proposed the Combined Helmhotz Integral Equation Formulation (CHIEF) [56] that alleviates the effect of the fictitious resonances by employing a certain number $N_{C H}$ of overdetermination points (CHIEF points) in the interior of the cavity that defines the exterior problem.

365 In essence, the CHIEF takes advantage of the lack of acoustic field inside the considered cavity to assemble $N_{C H}$ additional equations and transforms the 
square linear system (7) into the overdetermined linear system,

$$
\left[\begin{array}{c}
\mathbf{A}(\omega) \\
\mathbf{A}_{C H}(\omega)
\end{array}\right] \boldsymbol{\xi}(\omega)=\left[\begin{array}{c}
\mathbf{b}(\omega) \\
\mathbf{b}_{C H}(\omega)
\end{array}\right] \Longleftrightarrow \mathbf{A}_{A u g}(\omega) \boldsymbol{\xi}(\omega)=\mathbf{b}_{A u g}
$$

that can be solved in a least squares fashion. In the system of $(18), \mathbf{A}_{C H} \in$ $\mathbb{C}^{N_{C H} \times N}$ and $\mathbf{b}_{C H} \in \mathbb{C}^{N_{C H}}$ represent the system coefficients and right handside yielded through the same procedure equations $(6)$ and $(7)$ are derived, by deploying equation (2) for the preselected CHIEF points.

Employing the same series expansion of the kernel, equation 18 can be approximated analogously to equation (11) by simply substituting square derivative matrices $\mathbf{A}_{m}$ with $\mathbf{A}_{A u g, m}$, where the latter represent the derivative matrices necessary in the approximation of the augmented system $\mathbf{A}_{\text {Aug }}$.

In the context of model order reduction dealing with an overdetermined system of equations demands for a variation of the projection procedure, as the conjugate transpose of the right projection matrix $\mathbf{V}_{\ell}^{*}$ mismatches in dimensions with the system matrix dimensions. Therefore, in this case a different left projection matrix $\mathbf{W}_{A u g} \in \mathbb{C}^{\ell \times\left(N+N_{C H}\right)}$ needs to be employed leading to a Petrov-Galerkin or two sided projection. The left projection matrix $\mathbf{W}_{A u g}$ is constructed as

$$
\mathbf{W}_{\text {Aug }}=\left[\begin{array}{cc}
\mathbf{V}_{\ell}^{*} & 0 \\
0 & \mathbf{I}_{N_{C H} \times N_{C H}}
\end{array}\right]
$$

where $\mathbf{I}_{N_{C H} \times N_{C H}}$ is the identity matrix of dimension $N_{C H}$. Utilizing the left projection matrix $\mathbf{W}_{A u g}$ implies that no reduction is leveraged in the row space of the full system CHIEF coefficients $\mathbf{A}_{C H}$ or, analogously to the previous notation, of the derivative matrices $\mathbf{A}_{C H, m}$. Nevertheless, as usually a limited number of CHIEF points, e.g. $5 \%$ of the DOFs, is employed to stabilize the system, maintaining the dimension $N_{C H}$ without reduction is not considered detrimental for the proposed MOR technique. 


\section{Projection basis based on Krylov subspaces recycling}

The most significant part in Model Order Reduction techniques is usually the basis defined through the projection matrix $\mathbf{V}_{\ell}$, as this drives the quality of the reduced model. In this section, a method for constructing an appropriate basis is proposed employing Krylov subspaces.

\subsection{Assembling the projection basis}

The proposed technique is based on the recycling of Krylov subspaces for BEM systems at different frequencies. In detail, it consists of expanding Krylov subspaces for BEM systems on a grid of predefined master frequencies and collecting them to produce an appropriate basis for the reduced system. Consequently, the larger subspace that is generated, contains the subspace describing the first Ritz vectors of all BEM systems on the master frequencies' grid.

The Krylov subspaces can be represented by

$$
K_{q}^{f_{i}}\left(\mathbf{A}_{f_{i}}, \mathbf{b}_{f_{i}}\right)=\operatorname{span}\left\{\mathbf{b}_{f_{i}}, \mathbf{A}_{f_{i}} \mathbf{b}_{f_{i}}, \mathbf{A}_{f_{i}}^{2}, \mathbf{b}_{f_{i}}, \ldots, \mathbf{A}_{f_{i}}^{q-1} \mathbf{b}_{f_{i}}\right\} .
$$

where $f_{i}$ constitutes a single frequency of the master frequency grid. The order of the Krylov subspaces $q$ indicates the number of Ritz vectors of the BEM system that lie within the specific subspaces.

The Krylov subspaces of 20 are generated through an Arnoldi algorithm omitting the calculation of the upper Hessenberg matrix [29] (Algorithm 2). In order to prevent numerical instabilities, orthogonality is enforced amongst the Krylov subspaces through the Modified Gram-Schmidt procedure 61].

Having generated the Krylov subspaces $K_{q}^{f_{i}}$ up to a predefined order $q$ for all BEM systems of the master frequency grid, a larger subspace is devised containing all these Krylov subspaces as

$$
K_{\text {tot }}=\operatorname{span}\left\{K_{q}^{f_{1}} \cup K_{q}^{f_{2}} \cup \cdots \cup K_{q}^{f_{L}}\right\} .
$$

The projection basis $\mathbf{V}_{\ell}$ is constructed by orthogonalizing the collection of subspaces $K_{\text {tot }}$ as in 22 and truncating $\mathbf{U} \in \mathbb{C}^{N \times q L}$ with respect to the 


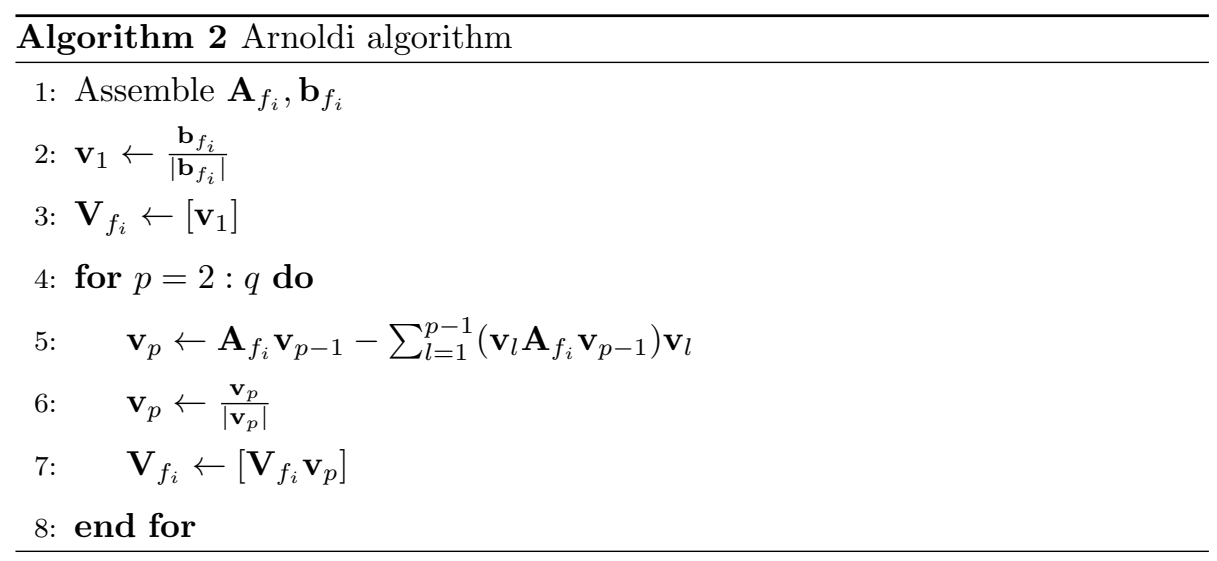

components of highest energy given by $\Sigma$ as

$$
\mathbf{U} \boldsymbol{\Sigma} \mathbf{V}^{T}=\operatorname{svd}\left(\left[\mathbf{V}_{f_{1}} \mathbf{V}_{f_{2}} \ldots \mathbf{V}_{f_{L}}\right]\right)
$$

and

$$
\mathbf{V}_{\ell}=\left[\mathbf{u}_{1} \mathbf{u}_{2} \ldots \mathbf{u}_{k}\right]
$$

in which, $\mathbf{u}_{i}$ represents a column vector of $\mathbf{U}$ and $\mathbf{u}_{k}, k<q L$ is the $k^{\text {th }}$ largest energy vector.

\subsection{Discussion about the projection basis}

Utilizing the projection matrix $\mathbf{V}_{\ell}$, the quality of the reduced order model achieved through expression (17) is high due to two main reasons. First, the BEM system at frequency $f_{x}$, a frequency line between two master frequencies $f_{i}$ and $f_{i+1}$, is projected on Krylov subspaces produced by BEM systems not only at these master frequencies but also at all other master frequencies. Equivalently, it is stated that the Krylov subspaces of all master frequencies are recycled for all the BEM systems of the frequency range under consideration. Thus, the subspaces, upon which the approximated eigenvectors (Ritz vectors) of BEM systems at the master frequencies lie, are exploited to project the BEM system at the in-between frequency $f_{x}$.

As Krylov subspaces recycling is deployed usually for slowly varying systems, the Krylov subspaces of the neighbouring master frequencies $f_{i}$ and $f_{i+1}$ will 
contribute the most to the expression of the solution subspace for the system at frequency $f_{x}$. However and secondly, the subspaces of Ritz vectors of BEM systems that lie further away in the frequency range are leveraged and their contribution is also important. Specifically, the Ritz vectors of a BEM system at a master frequency $f_{m} \approx 2 f_{x}$ will yield a surface variation of the acoustic variable of half the wavelength approximately, comparing to the Ritz vectors of the systems at adjacent master frequencies. Hence, a basis including the subspaces defined by the former Ritz vectors, i.e. $\mathbf{V}_{f_{m}}$, attributes more flexibility to the projected system and thus this adjusts better to the local differences occurring due to the recycling of the Krylov subspaces.

In the process of constructing the reduction basis, the two parameters that affect its quality are the location of the master frequencies and the order of the Krylov expansion. In this work, the master frequencies are selected according to a pre-defined frequency grid and the order of Krylov subspaces is maintained constant for all the master frequencies. Nevertheless, this selection does not reflect to the optimal settings of the MOR technique. Instead, considering the increasing importance of Krylov subspaces of higher frequencies' systems for the flexibility of the basis at the lower frequencies, it is more optimal to select e.g. a logarithmic distribution of the master frequencies in combination with an adaptive order for the assembled Krylov subspaces.

Finally, employing a multi-point Taylor expansion using meshes of different refinement at different regions of the frequency spectrum, as proposed in section 3.2, implies the construction of different projection matrices for each expansion. Nevertheless, constructing additional projection bases does not lead to additional computational cost as compared to constructing one single global basis. As the method is based on the Krylov subspace recycling, each basis will contain only the Krylov subspaces of the neighbouring master frequencies, resulting in constructed projection bases of lower order than the one yielded by a single point Taylor expansion.

460

Although, a multi-point Taylor expansion might seem preferable in terms of computational cost of constructing the bases and numerical stability of the 
polynomial expansion itself, the quality of the different bases might drop. As each basis will contain only the Krylov subspaces of the neighbouring master frequencies, the influence of the Krylov subspaces generated by higher frequency systems -having shorter wavelength variations- will be weakened or even cancelled.

\section{Error Estimator}

Exploiting the fact that the expanded Krylov subspaces span the subspaces of the approximated eigenvectors of the BEM systems, the definition of an error estimator is enabled. Specifically, Krylov subspaces of higher order, developed by a BEM system at a single master frequency, will match respectively the subspaces of a larger number of approximated eigenvectors of that system. Analogously, by increasing the order of Krylov subspaces at all master frequencies, subspaces of additional system eigenvectors are approximated also

475 for non-master frequencies systems. Thus, since the Krylov subspaces of order $q$ will be contained in the Krylov subspaces of order $q+1$ as illustrated by

$$
K_{q} \subseteq K_{q+1},
$$

a basis constructed by increasing the order of Krylov subspaces at all master frequencies will be more representative.

The true error induced by substituting the full scale BEM system by a Reduced Order Model (ROM) can be defined in (25) by the Euclidean norm of the difference of the approximated surface variables $\hat{\boldsymbol{\xi}}_{q}$ due to projection on the $K_{q}$, with the precise surface variables $\boldsymbol{\xi}$, normalized with the approximated quantities

$$
\epsilon_{q}=\frac{\left|\hat{\boldsymbol{\xi}}_{q}-\boldsymbol{\xi}\right|}{\left|\hat{\boldsymbol{\xi}}_{q}\right|}=\frac{\theta_{q}}{\left|\hat{\boldsymbol{\xi}}_{q}\right|} .
$$

Manipulating the right hand side of (25), an upper error bound for $\epsilon_{q}$ can 
be achieved by deploying the triangle inequality as

$$
\begin{aligned}
\epsilon_{q} & =\frac{\left|\hat{\boldsymbol{\xi}}_{q}-\boldsymbol{\xi}\right|}{\left|\hat{\boldsymbol{\xi}}_{q}\right|} \\
& =\frac{\left|\hat{\boldsymbol{\xi}}_{q}-\hat{\boldsymbol{\xi}}_{q+t_{1}}+\hat{\boldsymbol{\xi}}_{q+t_{1}}-\cdots-\hat{\boldsymbol{\xi}}_{q+t_{n}}+\hat{\boldsymbol{\xi}}_{q+t_{n}}-\boldsymbol{\xi}\right|}{\left|\hat{\boldsymbol{\xi}}_{q}\right|} \\
& \leq \frac{\left|\hat{\boldsymbol{\xi}}_{q}-\hat{\boldsymbol{\xi}}_{q+t_{1}}\right|}{\left|\hat{\boldsymbol{\xi}}_{q}\right|}+\cdots+\frac{\left|\hat{\boldsymbol{\xi}}_{q+t_{n-1}}-\hat{\boldsymbol{\xi}}_{q+t_{n}}\right|}{\left|\hat{\boldsymbol{\xi}}_{q}\right|}+\frac{\left|\hat{\boldsymbol{\xi}}_{q+t_{n}}-\boldsymbol{\xi}\right|}{\left|\hat{\boldsymbol{\xi}}_{q}\right|} \\
& =\frac{\theta_{q, q+t_{1}}}{\left|\hat{\boldsymbol{\xi}}_{q}\right|}+\cdots+\frac{\theta_{q+t_{n-1}, q+t_{n}}}{\left|\hat{\boldsymbol{\xi}}_{q}\right|}+\frac{\theta_{q+t_{n}}}{\left|\hat{\boldsymbol{\xi}}_{q}\right|},
\end{aligned}
$$

where $q<q+t_{1}<\cdots<q+t_{n}$. Considering that the subspace $K_{q+t_{n}}$ is more detailed, the norm of difference of the approximated solution to the true solution $\theta_{q+t_{n}}$ is assumed to be negligible and thus, it is truncated. This assumption is valid when $\theta_{q+t_{n-1}, q+t_{n}}$ takes low values as the convergence of the approximated solution has been initiated. In cases $\theta_{q+t_{n-1}, q+t_{n}}$ takes higher values, the assumption is not valid any more and the error estimator might fail to approximate the true error. Nevertheless, in such cases it is already indicated that the reduced model is not accurate enough by the non truncated terms of the sum in expression 26. Consequently, the error estimate can be formulated as follows,

$$
\epsilon_{q}=\frac{\left|\hat{\boldsymbol{\xi}}_{q}-\boldsymbol{\xi}\right|}{\left|\hat{\boldsymbol{\xi}}_{q}\right|} \leq \frac{\theta_{q, q+t_{1}}}{\left|\hat{\boldsymbol{\xi}}_{q}\right|}+\cdots+\frac{\theta_{q+t_{n-1}, q+t_{n}}}{\left|\hat{\boldsymbol{\xi}}_{q}\right|} .
$$

Relating to the BEM-MOR procedure, the derived error bound estimator implies that the full scale derivative matrices $\mathbf{A}_{m}$ of 11 , apart from being projected on the basis resulting from $K_{q}$, need to be projected as well on more detailed bases given by $K_{q+t_{1}}, \ldots, K_{q+t_{n}}$. Depending on how conservative the error bound estimator needs to be, one or more additional higher detailed projection bases are employed respectively. 


\section{Numerical assessment}

In this section, the proposed technique is first assessed in terms of algorithmic complexity and then it is deployed to accelerate two fast frequency sweep examples. The first problem is related to an exterior cube problem with the top surface vibrating while the second provides solution to a more industrially relevant problem, namely the interior acoustic response of a car cavity due to a monopole excitation. Without loss of generality, both examples are based on a single point Taylor expansion.

The method proposed in this work was developed in a Matlab environment based on the OpenBEM code provided by Henriquez et al. 62. The mesh of the geometries was generated through the Gmsh [63] and NX software. All computations are performed on a single machine of $32 \mathrm{~GB}$ RAM and $2.9 \mathrm{GHz}$ processing power.

\subsection{Theoretical algorithmic complexities}

The advantage of the proposed method emanates from the fact that most operations are shifted in the beginning of the frequency sweep and thus, the corresponding computational demands are transformed to fixed overhead costs i.e. off-line costs. These resources that are spent in advance, as demonstrated in

20 Table 1] are then compensated through the significantly reduced costs necessary per frequency that are provided in Table 2

Specifically, the parameters that affect the overhead costs, as emerge from equations (10)-23), can be identified as the order of the Taylor expansion $M_{\max }$, the size of the initial model $N$ and the size of the reduced model $\ell$, the number of master frequencies $L$ and the order of the Krylov expansion $q$.

The memory required for the off-line procedures apart from the assembly of the master frequencies' systems, illustrated in Table 1. considers a column by column assembly and projection of the Taylor matrices. Upon construction of each column, left projection occurs leading to memory requirements scaling with $\mathcal{O}(\ell \times N)$ for the storage of the two projection matrices and the derivative matrix under projection. The $\mathcal{O}\left(N^{2}\right)$ storage requirement of the master 


\begin{tabular}{|c|c|c|}
\hline Operation & Algorithmic efficiency & Required memory \\
\hline Assembly of Taylor matrices & $\mathcal{O}\left(M_{\max } \times N^{2}\right)$ & $\mathcal{O}(N)$ \\
Master frequencies system assembly & $\mathcal{O}\left(L \times N^{2}\right)$ & $\mathcal{O}\left(N^{2}\right)$ \\
Subspaces computation & $\mathcal{O}\left(L \times q \times N^{2}\right)$ & $\mathcal{O}(L \times q \times N)$ \\
Orthogonalization of subspaces & $\mathcal{O}\left(L^{2} \times q^{2} \times N\right)$ & $\mathcal{O}(L \times q \times N)$ \\
Projection of Taylor matrices & $\mathcal{O}\left(M_{\max } \times \ell \times N^{2}\right)$ & $\mathcal{O}(\ell \times N)$ \\
\hline
\end{tabular}

Table 1: Theoretical algorithmic efficiency and storage requirements of off-line operations

frequency system assembly might become a limitation in large industrial cases. However, in these cases instead of utilizing a conventional BEM system assembling procedure, an FMM-BEM or H-matrices algorithm can be employed to relax this storage requirement.

As can be deduced from Table 1 in order for the proposed method to be computationally more competitive than the conventional BEM, the following conditions must hold:

1. $\ell<N$

2. $M_{\max }<N_{f}$

3. $M_{\max } \times \ell^{2} \leq N^{2}$.

The first condition indicates that the order of the reduced model needs to be lower than the original model and describes the essence of model order reduction. The second is related to the number of the system or derivative matrix assemblies involved in the MOR technique, which is required to be lower than the total number of frequencies under consideration. The last condition is related to maintaining at most the same levels of memory as in a conventional BEM procedure. It indicates that the total memory required to store all the reduced SEBEM derivative matrices need to scale more favourably than storing one single full-scale model. Although in problems that are not memory demanding the latter need not be strictly satisfied, satisfying the two former remains crucial to maintain the computational advantage in terms of the total CPU time 
of the frequency sweep procedure. In fact, higher accelerations are achieved in case these are fulfilled in a more pronounced way.

\begin{tabular}{|c|c|c|c|}
\hline Operation & BEM & SEBEM & MOR \\
\hline Assembly of single matrix & $\mathcal{O}\left(N^{2}\right)$ & $\mathcal{O}\left(N^{2}\right)$ & $\mathcal{O}\left(\ell^{2}\right)$ \\
Solution of system & $\mathcal{O}\left(N^{3}\right)$ & $\mathcal{O}\left(N^{3}\right)$ & $\mathcal{O}\left(\ell^{3}\right)$ \\
\hline
\end{tabular}

Table 2: Comparison of theoretical algorithmic efficiency and storage requirements of online operations for BEM, SEBEM and MOR

The algorithmic efficiencies provided in Table 2 consider a direct system solution algorithm. Employing an iterative scheme for the respective procedure transforms the scaling of the system solution to $\mathcal{O}\left(s_{B E M} \times N^{2}\right), \mathcal{O}\left(s_{B E M} \times N^{2}\right)$ and $\mathcal{O}\left(s_{M O R} \times \ell^{2}\right)$ for BEM, SEBEM and MOR respectively, where $s_{B E M}$ and $s_{M O R}$ are the number of iterations to reach convergence of the solution for the full and reduced system, respectively.

\subsection{Cube Exterior problem}

The geometry of the first model investigated is depicted in Figure 3a. The model consists of a cube of $1 \mathrm{~m}$, located at the origin and towards the positive side of all axes. The lateral and bottom faces are assigned with the homogeneous Neumann boundary condition i.e. $u_{n}=0$, while the top surface is vibrating with $u_{n}=1 \frac{m}{s}$.

The mesh demonstrated in Figure $3 \mathrm{~b}$ is composed of 2606 nodes and 5208 triangular elements. The validity of the mesh extends up to $1200 \mathrm{~Hz}$ considering 6 elements per wavelength, the minimum requirement as reported in [12. To the yielded square system of $N=2606$ DOFs, additional 130 CHIEF points equations are appended, resulting in a $2736 \times 2606$ overdetermined system.

The frequency range of interest extends on $F_{\text {range }}=[50,1200] \mathrm{Hz}$, while the system is solved with a $1 \mathrm{~Hz}$ increment. In terms of the non-dimensional Helmholtz number the range of interest is $k a_{\text {range }}=[0.9,22]$, where $a_{\text {range }}=1 \mathrm{~m}$ being the characteristic dimension of the geometry. Following a conventional 


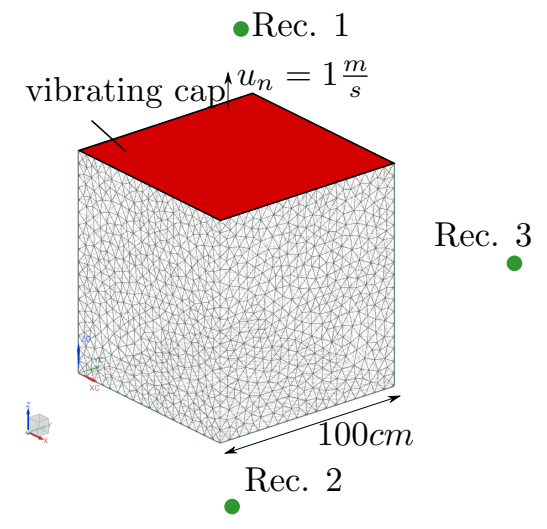

(a) Cube sketch

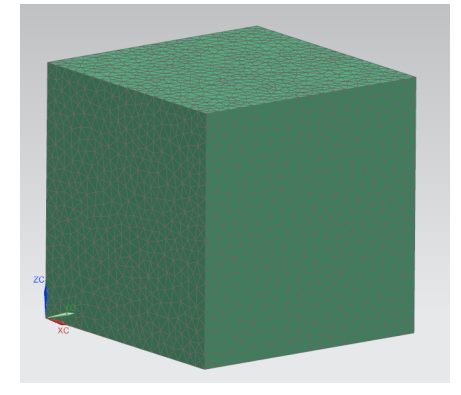

(b) Cube mesh

Figure 3: Exterior cube problem with vibrating cap

BEM procedure, this rises the needs to assembling and solving the system for 1151 distinct frequency lines.

\subsubsection{Model reduction}

According to the dimensions of the model, the maximum distance $r_{\max }$ as defined in 12 is the big diagonal of the cube, namely $r_{\max } \approx 1.73 \mathrm{~m}$. Consulting Figure 2, an expansion of the BEM kernel up to the $60^{\text {th }}$ order satisfies the minimum requirement for a maximum residual, $\sigma_{\max }=0.01$. In detail, the $60^{\text {th }}$ order expansion provides a maximum range of validity of $\Delta f_{\max } \approx 600 \mathrm{~Hz}$. Thus, selecting as an expansion point the middle frequency of the range under consideration i.e. $600 \mathrm{~Hz}$, renders the expansion valid for the whole range of interest. Additionally, a series expansion of order 60 satisfies the second condition of section 6.1 and thus, the computational advantage of the MOR technique is ensured.

In order to assemble the reduction subspace, the procedure described in section 4 is followed. Krylov subspaces are generated as expressed in 21. by the BEM systems constructed for a grid of master frequencies $f_{\text {master }}$. These are defined by frequency increments of $50 \mathrm{~Hz}$, taking the values $f_{\text {master }}=$ $[50,100, \ldots, 1150,1200]$. Subsequently, the collection of Krylov subspaces is or- 
thogonalized and truncated as in 222.

The construction of the projection matrix is conducted by employing Krylov subspaces of order 15 at each master frequency. Given the number of the master frequencies selected, the resulting union of these subspaces produces an $N \times 360$ matrix, which is orthogonalized and truncated for subspaces with energy less than $10^{-4}$. Finally, the yielded projection matrix is $\mathbf{V}_{\ell} \in \mathbb{C}^{N \times 306}$, leading to a reduced order model of 306dofs, namely $88 \%$ fewer than the full order model, satisfying the first condition stated in section 6.1. Including the CHIEF overdetermination equations, the system takes dimensions $436 \times 306$ and is solved in a least-square manner.

Inspecting the memory requirements of the reduced model, the third condition in section 6.1 indicates that the total memory of the SEBEM reduced matrices scales at least similarly to one full-scale model BEM matrix. In this problem this is translated by constructing a reduced order model that employs $\sim 1 / 7-1 / 8$ of the initial number of the degrees of freedom of the cube model, which is also fulfilled by the constructed reduced model.

Regarding the computation of the error estimate, one new, more enriched basis is created. In detail, this time, before orthogonalizing and truncating the collection of the Krylov subspaces, the order of the Krylov subspaces produced per master frequency is increased, to further enrich the projection basis. Specifically, referring to (27) it is selected $n=1$ and $t_{1}=4$ and thus, the basis is created by collecting the $19^{\text {th }}$ order Krylov subspaces, yielding a reduced model of 390DOFs.

\begin{tabular}{|c|c|c|c|}
\hline Evaluation points & $x$ & $y$ & $z$ \\
\hline Rec. 1 & $0.5 \mathrm{~m}$ & $0.5 \mathrm{~m}$ & $-0.5 \mathrm{~m}$ \\
Rec. 2 & $0.5 \mathrm{~m}$ & $0.5 \mathrm{~m}$ & $1.5 \mathrm{~m}$ \\
Rec. 3 & $1.5 \mathrm{~m}$ & $1.5 \mathrm{~m}$ & $0.5 \mathrm{~m}$ \\
\hline
\end{tabular}

Table 3: Coordinates of domain evaluation points; Cube model $(N=2606)$ with vibrating cap 

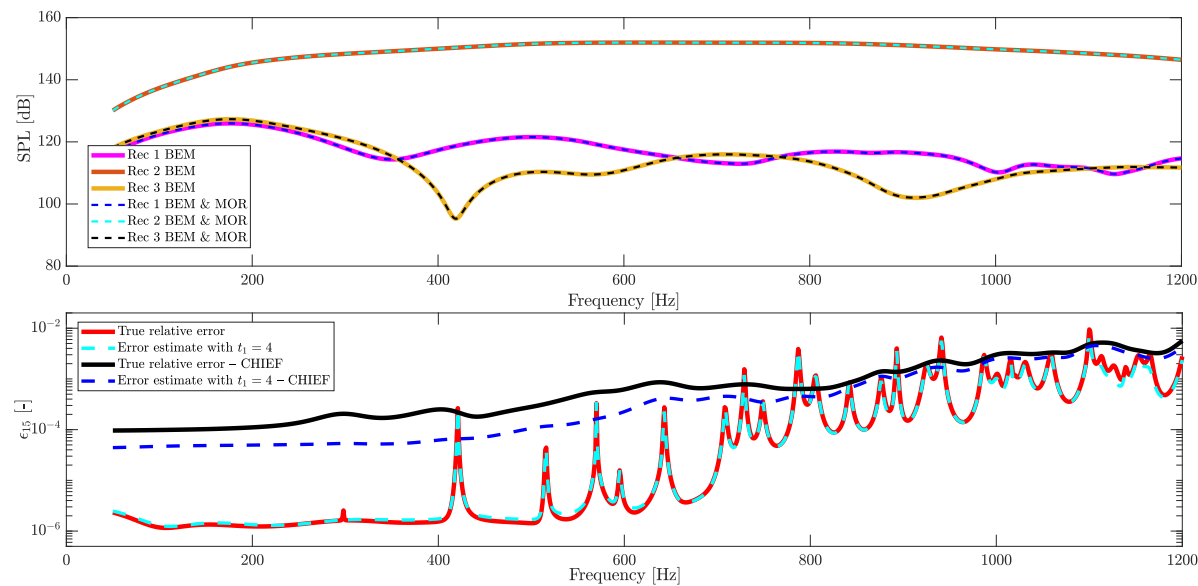

Figure 4: Sound Pressure Levels at evaluation points of Table 3 (top). True relative error $\epsilon_{15}$ and error estimate of surface pressure reduced model of 308dofs (bottom); Cube model with vibrating cap

In the following, the acoustic quantities yielded from OpenBEM code 62 are considered as benchmark results. The sound pressure levels are evaluated at 3 domain points, as given in Table 3 and illustrated in Figure 4 . In the same figure, the relative error and the relative error estimates that are induced to the surface pressure distribution by the proposed model reduction, are demonstrated both including and omitting CHIEF overdetermination.

The incurring error for both cases does not exceed the threshold of $1 \%$, rendering the ROM acceptable for the whole frequency range under consideration. However, the relative errors and error estimators for the two cases demonstrate different behaviours. Specifically, omitting CHIEF overdetermination results in rougher but of a lower magnitude error curves, while the error estimator observes closely the true relative error curve. On the contrary, the error curves including CHIEF treatment are smoother but overall associated with higher values of error, while the error estimator deviates from the true error curve. This different behaviour is, on the one hand, a consequence of failing to include information related to the row spaces of the CHIEF points to the constructed basis as elaborated in section 3.4. while on the other hand, the smoothness of 
the curves in the second case is connected with the treatment of the irregular frequencies. Although, in this case the relative error including CHIEF reflects the true error of the model reduction technique, the curves related to the nonCHIEF procedure are a more representative measure for the quality of the basis employed to project the full system.

Finally, the lower relative errors that are observed at lower frequencies can be associated with two facts. On the one hand, the surface pressure distribution at lower frequencies is usually simpler to describe, making the local recycling of fewer Krylov subpspaces more efficient. On the other hand, the subspaces spanned by the Ritz vectors of the system at higher frequencies contribute to describe inter-frequency local spatial variations of the surface pressures. In fact, referring to the non-CHIEF relative error curve, it can be visually divided into two distinct parts. The first part covers the region below $f_{\max } / 2=600 \mathrm{~Hz}$, where the projection space includes Ritz vectors of systems of at least double the frequency and thus, the error takes its lowest values, while in the second part above $600 \mathrm{~Hz}$, the error is inflated by some orders of magnitude. Thus, introducing additional Ritz vectors of higher frequencies systems would further reduce the error as well in the second part of the frequency range.

\subsubsection{Computational resources analysis}

Although through this frequency sweep analysis example the full potential acceleration of the proposed method is not pronounced, already a computational gain is apparent. Due to the small size of the model, the absolute accumulated computational cost saved is mostly attributed to the acceleration of the system assembly and less to the speed-up of the solution of the system. However, due to the cubic algorithmic complexity of the latter, it is expected that by an increase of the number of degrees of freedom, the absolute speed-up of that will take more effect.

In Figure 5, the required computational resources for producing the frequency sweep response of the considered model are illustrated, using conventional BEM, SEBEM and the proposed model reduction technique (labelled as 


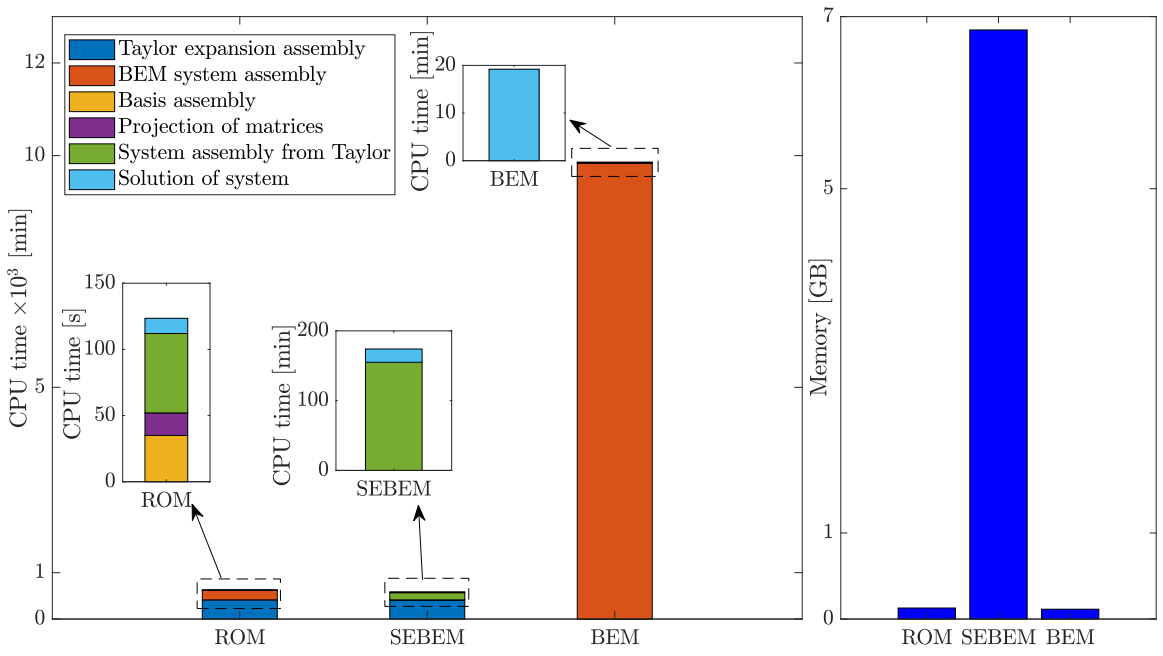

Figure 5: Computational resources comparison measured in CPU time and required memory; Cube model with vibrating cap

$\mathrm{ROM}$ ). Most of the CPU time utilized in all different techniques is associated with either the system assembly for conventional BEM or with the derivative matrices construction in the SEBEM and SEBEM \& MOR technique. Thus, based on the total CPU time needed for this model, the computational advantage of the MOR technique is not pronounced. Nevertheless, in relative terms, the model reduction technique still outperforms the SEBEM regarding the online operations, as the associated CPU time scales with a different order of magnitude $-[s]$ and $[\mathrm{min}]$ respectively.

The overall computational advantage of the proposed model reduction technique is evident in the right part of Figure 5 despite the small size of the model. Specifically, although the total necessary CPU time scales similarly to that of 675 SEBEM, comparing the storage costs of the two methods, the benefit offered by the proposed technique is clear, as in SEBEM all the full-scale derivative matrices need to be stored, inflicting a multiple storage cost.

As described in section 5 , the error estimator degenerates into a computation of an additional reduced model. Thus, the cost of the error estimator scales similarly to that of the construction of the original reduced model. 


\begin{tabular}{|c|c|c|}
\hline Operation & SEBEM \& MOR & Cost type \\
\hline Assembly of single Taylor matrix & $6 \mathrm{~m} 50 \mathrm{~s}$ & Off-line \\
System assembly per master frequency & $8 \mathrm{~m} 40 \mathrm{~s}$ & Off-line \\
Subspaces computation per master frequency & $1.5 \mathrm{~s}$ & Off-line \\
Orthogonalization of subspaces & $1 \mathrm{~s}$ & Off-line \\
Projection per Taylor matrix & $0.25 \mathrm{~s}$ & Off-line \\
Assembly of reduced system per frequency & $0.05 \mathrm{~s}$ & Online \\
Solution of reduced system per frequency & $0.01 \mathrm{~s}$ & Online \\
\hline
\end{tabular}

Table 4: Computational costs of all operations involved into Series expansion reduced BEM measured in CPU time; Cube model $(N=2606)$ with vibrating cap

In Table 4 the computational costs of the proposed method are illustrated along with the type of the cost. An off-line cost, in contrast to an online cost, is related to an action that is not performed for each frequency line under consideration, but rather in advance of the frequency sweep, to acquire the derivative matrices and the essential respective projection bases. The yielded CPU time for online costs exhibits insignificant values as the related operation scales now as a function of the number of degrees of freedom of the reduced system. On the contrary, as the off-line costs scale with the number of the DOFs of the full system, the off-line cost of constructing the reduced model is comparable to that of assembling a number of conventional BEM systems.

Consequently, the frequency sweep using the proposed technique becomes increasingly advantageous when the computation of systems at multiple frequency lines is involved. Thus, the higher the number of frequency lines the BEM solution is required, the more pronounced acceleration is expected as stated as well in section 6.1. Selecting a larger frequency increment of $5 \mathrm{~Hz}$ would signify a reduction of all online costs by a factor of 5 . Specifically, the CPU times of Figure 5 that are related to the number of frequency lines to be solved would be scaled down by the factor 5 . As a result the speed-up offered by the proposed technique would be less pronounced.

${ }_{700}$ Having constructed the reduced model, the speed-up factors offered by the 


\begin{tabular}{|c|c|c|c|}
\hline Operation & Cost & \multicolumn{2}{|c|}{ Speed-up factor } \\
\hline Method & Conventional BEM & SEBEM & SEBEM \& MOR \\
\hline $\begin{array}{c}\text { System assembly } \\
\text { System solution }\end{array}$ & $8 \mathrm{~m} \mathrm{40s}$ & 57.4 & $10.3 \times 10^{3}$ \\
\hline
\end{tabular}

Table 5: Comparison of computational costs of operations performed online between BEM, SEBEM and SEBEM \& MOR; Cube model $(N=2606)$ with vibrating cap

proposed technique for each frequency line are considerably high. As indicated in Table 5 in this case the more highlighted gain originates from the system assembly, however, taking into account the algorithmic efficiencies correlated to the system assembly and system solution $\left(\mathcal{O}\left(N^{2}\right)\right.$ and $\mathcal{O}\left(N^{3}\right)$ respectively), this will be reversed for larger models.

\subsubsection{Parameters affecting the model reduction efficiency}

As described in section 6.1, the number of master frequencies and the order of Krylov subspaces expanded at each master frequency constitute the most important parameters that affect the computational efficiency of the model order reduction technique. Although in this work a constant spacing of master frequencies and order of Krylov subspaces are utilized, in this section the influence of the choice of the frequency spacing and the order of Krylov subspaces is investigated.

In order to examine the efficiency of the reduced model the following quantities are considered: i) the total relative error over the frequency range $\epsilon=\left|\epsilon_{q}\right|_{2}$, where $q$ is the respective order of the Krylov subspaces, ii) the memory scaling comparing to the conventional BEM $\mu$ and iii) the acceleration rate of the online procedures $\alpha$ with respect to the necessary CPU time in the SEBEM procedure. Using these measures, both efficiency parameters $\alpha$ and $\mu$ are compared to the most competitive alternative method of the two benchmark methods. Specifically, although by the measure $\mu$ the required memory of the proposed technique is compared to the memory utilised in the conventional BEM, measure $\alpha$ reflects to the respective speed-up offered only by the projection on a reduced basis and 

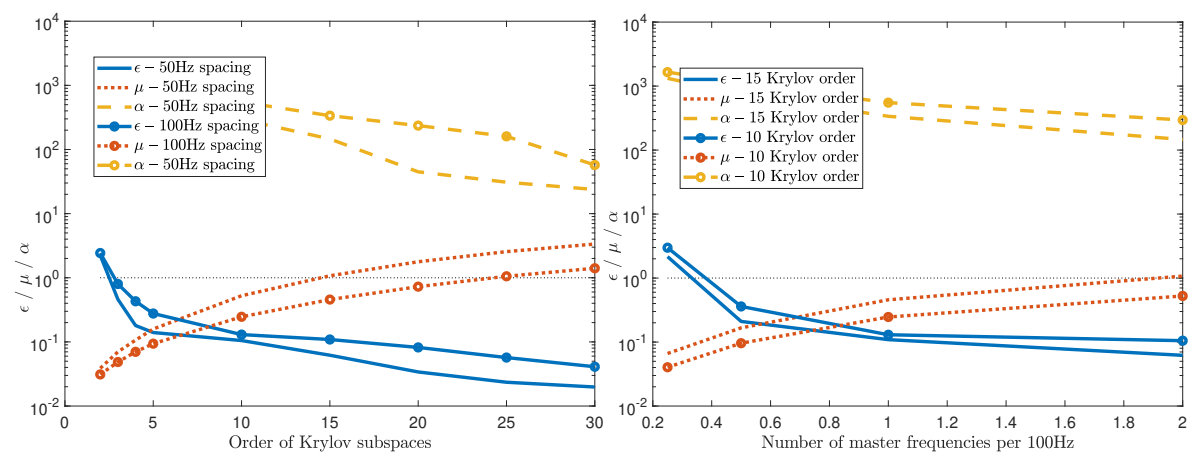

Figure 6: Total error $\epsilon$, memory scaling $\mu$ and acceleration rate $\alpha$ varying order of Krylov subspaces or number of master frequencies; Cube model with vibrating cap

not through the series expansion scheme.

In Figure 6 the accuracy together with the computational efficiency of the model reduction technique are depicted. As illustrated, all measures of computational efficiency scale similarly for increasing either the order of the Krylov subspaces or the density of the master frequencies grid. In both cases, the measures tend to asymptotically converge to a constant value. This behaviour is justified, as in the former case, appending additional Krylov subspaces does not contribute to the basis with subspaces yielded by systems of different wavenumbers, while in the latter case adding master frequencies of low order Krylov subspaces does not cover the subspace of enough approximated system eigenvectors, especially for the higher frequency range.

Considering this behaviour, the optimal subspaces could result through an adaptive procedure where the density of the master frequencies grid would increase in parallel with the order of the Krylov subspaces adaptively for the higher frequency regime. Thus, both additional subspaces that vary with different wavelength would be leveraged and the subspace of a sufficient number of eigenvectors would be matched, maintaining in the same time the order of the reduction basis in acceptable limits that respect condition 3 of section 6.1 . 


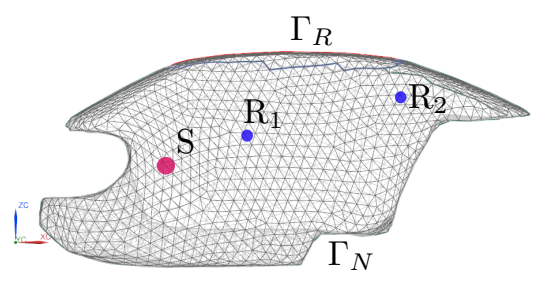

(a) Car cavity sketch

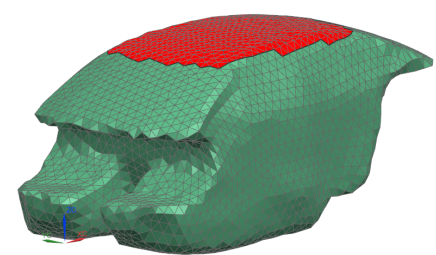

(b) Car cavity mesh and absorbing elements

Figure 7: Interior car cavity problem with absorbing roof and monopole excitation

\subsection{Car Cavity interior problem}

Next, the interior problem of a car cavity is evaluated. A car interior surface is considered, assigning constant impedance at the elements of roof of the car $Y_{0}=\frac{1}{\rho_{0} c}=0.0024 \mathrm{~kg} / \mathrm{m}^{2} s$ and the homogeneous Neumann condition $u_{n}=0$ for the rest of the elements. Monopole excitation is selected at position $S$ and the sound pressure is evaluated in two locations $R_{1}$ and $R_{2}$. The above information is graphically depicted in Figure $7 \mathrm{a}$.

The mesh of the model (Figure 7b) consists of 6500 elements leading to a number of nodes and DOFs of $N=3253$. Fulfilling the requirement of 6 elements per wavelength, the range of validity of the model reaches $700 \mathrm{~Hz}$. Taking an increment of $1 \mathrm{~Hz}$ the system assemblies and solutions required, amount to 651 frequency lines for the range of $F_{\text {range }}=[50,700]$ or in terms of non-dimensional Helmholtz number $k a_{\text {range }} \approx[2,33]$, with $a_{\text {range }}=2.2 \mathrm{~m}$.

\subsubsection{Model Reduction}

Considering the maximum distance separating any two elements $r_{\max }=$ $3.25 \mathrm{~m}$, a $50^{t h}$ order Taylor expansion of the BEM kernel is selected. Approximately the central frequency of the range of interest is elected as the expansion frequency, namely $f_{0}=400 \mathrm{~Hz}$.

Due to the complexity of the geometry and the nature of the interior problem, where several cavity resonances take place, a finer grid of master frequencies is utilized by taking a $25 \mathrm{~Hz}$ spacing and a higher order of Krylov subspaces is selected. Thus, the Arnoldi procedure (2) is deployed for $f_{\text {master }}=$ 
$[50,75, \ldots, 675,700]$, constructing 30-dimensional subspaces for each master frequency. Accumulating these subspaces and orthogonalizing, an orthogonal basis of order 810 is obtained. Truncating the subspaces of energy lower than $10^{-4}$, yields the final projection matrix $\mathbf{V}_{\ell} \in \mathbb{C}^{N \times 657}$ leading to a model with $80 \%$ fewer DOFs than the original model. The reduced model satisfies all the conditions stated in section 6.1, related to the parameters ranking the proposed technique as more competitive than the conventional BEM.

For the respective error estimator, a more elaborate subspace for each master frequency is employed, namely spanning 35 dimensions. By truncation of lowest energy vectors, the devised reduced model for the error estimator reaches 750 DOFs.
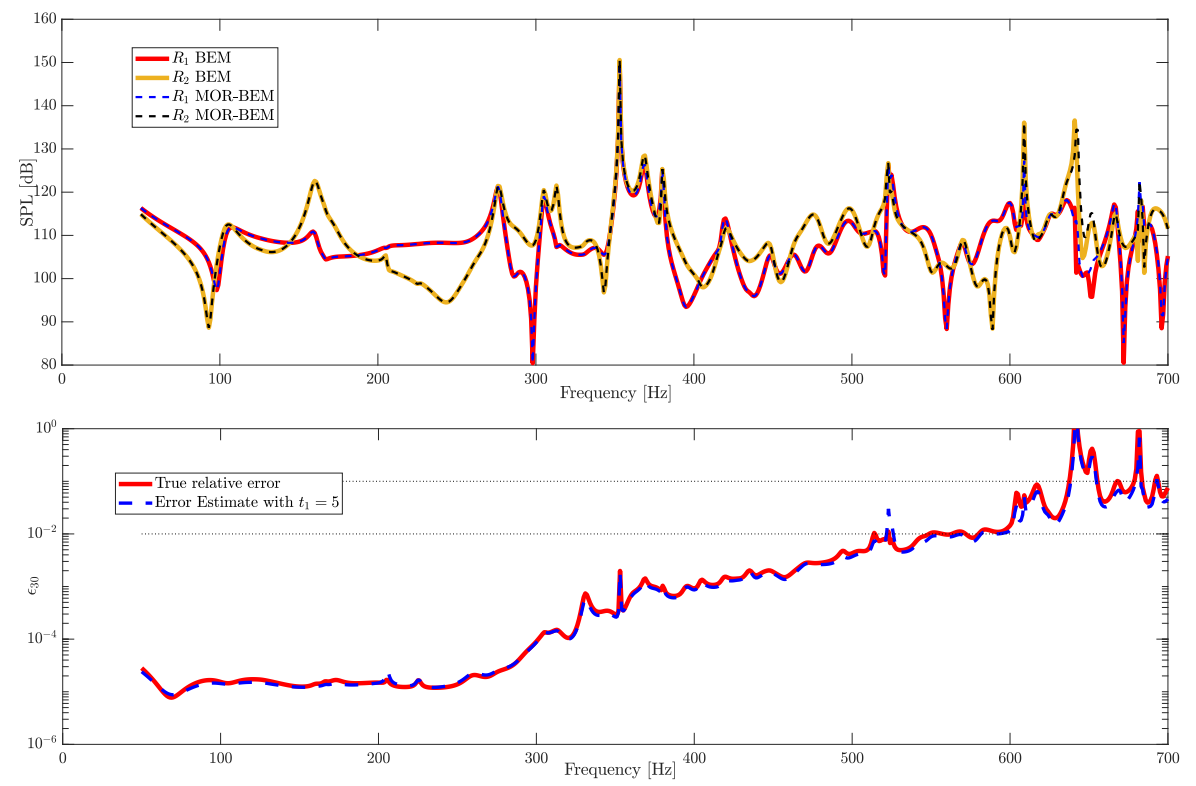

Figure 8: Sound Pressure Levels at $R 1$ and $R 2$ from car cavity sketch in 7 a (top). True relative error $\epsilon_{30}$ and error estimate of surface pressure reduced model of $657 \mathrm{dofs}$ (bottom); Interior car cavity problem with absorbing roof and monopole excitation

In Figure 8 , the sound pressure levels at receivers $R 1$ and $R 2$ are plotted, as well as the respective relative error for the surface pressure distribution. The acoustic quantities yielded from OpenBEM code 62 are considered as bench- 
mark results. The relative error satisfies the criterion of $1 \%$ for the region until $600 \mathrm{~Hz}$, while it increases for the rest of the frequency range under consideration. This increased error is attributed to the highly resonant behaviour of the cavity, as illustrated also in the graph of the computed sound pressure levels.

In general, in cases of highly resonant problems, the proposed model reduction scheme necessitates the use of more Ritz vectors of the corresponding BEM systems to describe accurately the acoustic response on the boundary. Thus, recycling of a higher number of Krylov subspaces needs to be performed on a denser master frequency grid. However, still for a relative error of around $10 \%$ the sound pressure levels coincide with a difference of $1 \mathrm{~dB}$.

The constructed error estimator succeeds in predicting the true relative error especially for the lower frequency region. In the higher frequencies where the true relative error demonstrates higher values, the error estimator fails to approximate so closely the true relative error curve. Due to the highly resonant behaviour of the model, the last term of estimated error bound that is truncated in expression (26) cannot be considered negligible. Nevertheless, although the error estimator does not predict the correct true error, it is predicted that the reduced model is not accurate enough in this frequency range.

To improve the accuracy of the reduced model in high frequencies, instead of deploying a fixed-spacing frequency grid with constant order of Krylov subspaces, an adaptive distribution of the master frequencies could be leveraged with varying order of Krylov subspaces. Taking into account the higher errors involved in the higher frequencies of the frequency interval under consideration, an adaptive approach would include a gradual densification of the master frequencies grid and an increasing order of the Krylov subspaces for the higher frequency region. However, this procedure needs to respect the constraints defined in section 6.1 to ensure the computational advantage.

\subsubsection{Computational resources analysis}

In Figure 9, the computational resources required by the three methods are illustrated as in the problem of section 6.2. Similarly to the cube exterior 
problem, due to the small size of the model, the most apparent computational advantage in terms of CPU time is related to the system assembly. In detail, both the proposed method (labelled as ROM) and the SEBEM offer an equivalent total computational speed-up as the assembly of discrete system matrices for all frequency lines is avoided. The SEBEM appears to be even faster in comparison with the proposed method, as it does not require the assembly of any BEM matrices apart from the Taylor expansion derivative matrices.

Nevertheless, as illustrated by the respective subfigures, the online operations performed by the proposed technique necessitate one order of magnitude less CPU time comparing to the SEBEM and conventional BEM. Additionally, the proposed model reduction scheme demonstrates superior behaviour in terms of storage required. Namely, a slightly higher amount of memory is necessitated for the whole frequency range of interest than the memory involved in the solution of a single frequency line with the conventional BEM.
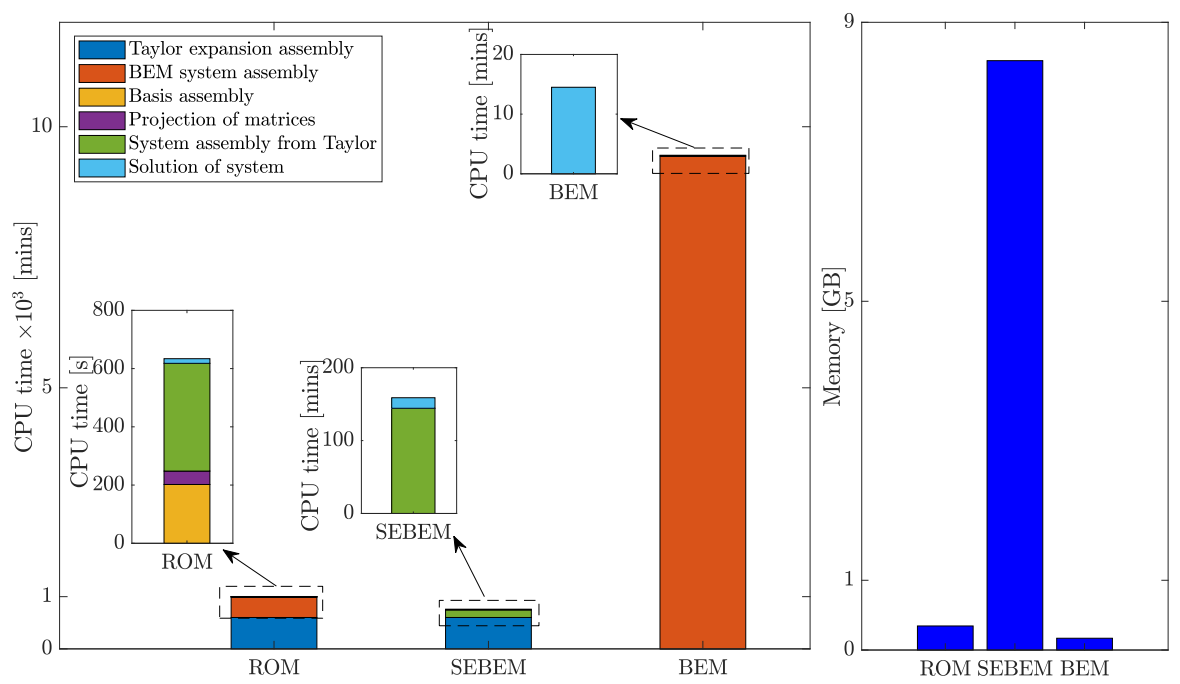

Figure 9: Computational resources comparison measured in CPU time and required memory; Interior car cavity problem with absorbing roof and monopole excitation

Generally, disregarding the cost of solving the system, the proposed method becomes more computationally efficient than the conventional BEM in case the 
number of frequencies assembling the BEM system is higher than the combined

\begin{tabular}{|c|c|c|c|}
\hline Operation & Cost & \multicolumn{2}{|c|}{ Speed-up factor } \\
\hline Method & Conventional BEM & SEBEM & SEBEM \& MOR \\
\hline System assembly & $14 \mathrm{~m} 30 \mathrm{~s}$ & 72.5 & 1450 \\
System solution & $1.3 \mathrm{~s}$ & 1 & 53.6 \\
\hline
\end{tabular}

Table 6: Comparison of computational costs of operations performed online between BEM, SEBEM and SEBEM \& MOR; Interior car cavity problem with absorbing roof and monopole excitation

\section{Conclusions}

In this work, a novel model reduction technique for the direct collocational Boundary Element Method is introduced in the context of linear acoustics. The 
proposed technique accelerates in particular the frequency sweep analysis for a specific BEM problem. A series expansion of the Green's function is leveraged to express the BEM system as a series of frequency-decoupled matrices. Subsequently, these matrices are reduced by a Galerkin one-sided projection to economize on both memory and algorithmic efficiency. The basis upon which the projection is performed, is yielded by spanning the subspace of the Ritz vectors produced on a master frequency grid. The procedure is arranged algorithmically in such a manner that the memory requirements of the method scale more favourably to that of a conventional BEM procedure. Due to the overhead cost of providing the Taylor derivative matrices and constructing the corresponding projection basis, the proposed method is especially suitable for frequency sweep analyses, as the off-line cost needs to be compensated with the computational speed-up gained through the online operations. Finally, an error estimator is employed to assess the quality of the produced reduced model.

The present work constitutes a proof of concept for the combination of the model order reduction with the BEM. The Taylor expansion selected for the frequency decoupling offers the advantage of analytical calculation of the polynomial coefficient of the constructed series, however, the respective procedure is quite intrusive comparing to current methods. This problem can be addressed using alternative polynomial expansion techniques. Additionally, the use of the direct BEM is not mandatory, as the generality of the polynomial expansion can offer similar techniques for the indirect BEM formulation and an extension of the direct method with a Burton-Miller approach [55]. These suggestions form the basis for future improvements to the proof of concept as presented in this paper.

870 Finally, in the paper the efficiency of the method is demonstrated by tackling a simple and a more industrially relevant example. Significant speed-up factors have been reported both for the assembly and the solution of the BEM system, maintaining in parallel low relative errors. The generated error estimator shows in general good agreement with the true relative error, indicating robustly the quality of the reduced model. As illustrated through the examples, the method 
is more efficient for less complex and less resonant geometries as recycling of Krylov subspaces of lower dimensions is required to ensure an acceptable relative error. This renders the method more applicable for exterior problems, where usually less resonances occur.

\section{Acknowledgements}

The research of D. Panagiotopoulos is funded by an Early Stage Researcher grant within the European Project PBNv2 Marie Curie Initial Training Network (GA 721615) and the research of E. Deckers is funded by a grant from the Research Foundation - Flanders (FWO). Additionally, the Research Fund KU

Leuven is gratefully acknowledged for its support.

\section{References}

[1] C. A. Brebbia, The boundary element method for engineers, Pentech press, 1980.

[2] P. K. Banerjee, R. Butterfield, Boundary element methods in engineering science, Vol. 17, McGraw-Hill London, 1981.

[3] M. Costabel, Principles of boundary element methods, Techn. Hochsch., Fachbereich Mathematik, 1986.

a [4] C. Brebbia, R. Butterfield, Formal equivalence of direct and indirect boundary element methods, Applied Mathematical Modelling 2 (2) (1978)

895 132 - 134. doi:https://doi.org/10.1016/0307-904X (78)90052-5.

URL http://www.sciencedirect.com/science/article/pii/ $0307904 \times 78900525$

[5] G. Rieckh, W. Kreuzer, H. Waubke, P. Balazs, A 2.5 d-fourier-bem model for vibrations in a tunnel running through layered anisotropic soil, Engineering Analysis with Boundary Elements 36 (6) (2012) 960-967. 
[6] M. Bonnet, G. Maier, C. Polizzotto, Symmetric galerkin boundary element methods, Applied Mechanics Reviews 51 (11) (1998) 669-704.

[7] S. Marburg, B. Nolte, Computational acoustics of noise propagation in fluids: finite and boundary element methods, Vol. 578, Springer, 2008.

[8] P. Bettess, Infinite elements, International Journal for numerical methods in engineering 11 (1) (1977) 53-64.

[9] Q. Qi, T. L. Geers, Evaluation of the perfectly matched layer for computational acoustics, Journal of Computational Physics 139 (1) (1998) 166-183.

[10] Q.-H. Liu, J. Tao, The perfectly matched layer for acoustic waves in absorptive media, The Journal of the Acoustical Society of America 102 (4) (1997) 2072-2082.

[11] I. Harari, T. J. Hughes, A cost comparison of boundary element and finite element methods for problems of time-harmonic acoustics, Computer Methods in Applied Mechanics and Engineering 97 (1) (1992) 77-102.

[12] S. Marburg, Six boundary elements per wavelength: is that enough?, Journal of Computational Acoustics 10 (01) (2002) 25-51.

[13] M. Fischer, U. Gauger, L. Gaul, A multipole galerkin boundary element method for acoustics, Engineering analysis with boundary elements 28 (2) (2004) 155-162.

[14] Y. Liu, N. Nishimura, The fast multipole boundary element method for potential problems: a tutorial, Engineering Analysis with Boundary Elements 30 (5) (2006) 371-381.

[15] W. Hackbusch, B. N. Khoromskij, A sparse h-matrix arithmetic, Computing 64 (1) (2000) 21-47.

925 [16] S. M. Kirkup, D. Henwood, Methods for speeding up the boundary element solution of acoustic radiation problems, Journal of vibration and acoustics 114 (3) (1992) 374-380. 
[17] H. Schenck, G. Benthien, The application of a coupled finite-element boundary-element technique to large-scale structural acoustic problems, Advances in boundary elements 2 (1989) 309-317.

[18] G. W. Benthien, H. Schenck, Structural-acoustic coupling, in: Boundary element methods in acoustics, Computational Mechanics Publications Southampton, UK, 1991, pp. 109-129.

[19] T. Wu, W. Li, A. Seybert, An efficient boundary element algorithm for multi-frequency acoustical analysis, The Journal of the Acoustical Society of America 94 (1) (1993) 447-452.

[20] G. H. Koopmann, H. Benner, Method for computing the sound power of machines based on the helmholtz integral, The Journal of the Acoustical Society of America 71 (1) (1982) 78-89.

[21] Z. Wang, Z. Zhao, Z. Liu, Q. Huang, A method for multi-frequency calculation of boundary integral equation in acoustics based on series expansion, Applied Acoustics 70 (3) (2009) 459-468.

[22] Q. Zhang, Y. Mao, D. Qi, Y. Gu, An improved series expansion method to accelerate the multi-frequency acoustic radiation prediction, Journal of Computational Acoustics 23 (01) (2015) 1450015.

[23] J.-S. Oh, S.-K. Youn, An efficient technique for the indirect bem for multifrequency acoustic analysis using green's function approximation, Journal of Mechanical Science and Technology 32 (5) (2018) 1965-1973.

[24] S. Lefteriu, M. S. Lenzi, H. Beriot, M. Tournour, W. Desmet, Fast frequency sweep method for indirect boundary element models arising in acoustics, Engineering Analysis with Boundary Elements 69 (2016) 32-45.

[25] R. D. Slone, R. Lee, J.-F. Lee, Broadband model order reduction of polynomial matrix equations using single-point well-conditioned asymptotic waveform evaluation: derivations and theory, International Journal for Numerical Methods in Engineering 58 (15) (2003) 2325-2342. 
[26] R. D. Slone, R. Lee, J.-F. Lee, Well-conditioned asymptotic waveform evaluation for finite elements, IEEE Transactions on Antennas and Propagation 51 (9) (2003) 2442-2447.

[27] Z. Bai, Krylov subspace techniques for reduced-order modeling of largescale dynamical systems, Applied numerical mathematics 43 (1-2) (2002) $9-44$.

[28] E. J. Grimme, KRYLOV PROJECTION METHODS FOR MODEL REDUCTION, Tech. rep. (1992). URL https://perso.uclouvain.be/paul.vandooren/ThesisGrimme. pdf

[29] A. C. Antoulas, Approximation of large-scale dynamical systems, Vol. 6, Siam, 2005.

[30] J. Wolf, Modal synthesis for combined structural-acoustic systems, AIAA Journal 14 (1) (1976) 33-38.

[31] S. H. Sung, D. J. Nefske, Component mode synthesis of a vehicle structuralacoustic system model, AIAA journal 24 (6) (1986) 1021-1026.

[32] S. M. Kirkup, S. Amini, Solution of the helmholtz eigenvalue problem via the boundary element method, International Journal for Numerical Methods in Engineering 36 (2) (1993) 321-330.

975 [33] A. Chatterjee, An introduction to the proper orthogonal decomposition, Current science (2000) 808-817.

[34] Y. Liang, H. Lee, S. Lim, W. Lin, K. Lee, C. Wu, Proper orthogonal decomposition and its applications - part i: Theory, Journal of Sound and vibration 252 (3) (2002) 527-544.

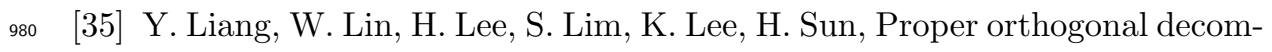
position and its applications-part ii: Model reduction for mems dynamical analysis, Journal of Sound and Vibration 256 (3) (2002) 515-532. 
[36] F. Chinesta, P. Ladevèze, Separated representations and pgd-based model reduction, Fundamentals and Applications, International Centre for Mechanical Siences, Courses and Lectures 554 .

[37] Y. Saad, M. H. Schultz, Gmres: A generalized minimal residual algorithm for solving nonsymmetric linear systems, SIAM Journal on scientific and statistical computing 7 (3) (1986) 856-869.

[38] S. Marburg, S. Schneider, Performance of iterative solvers for acoustic problems. part i. solvers and effect of diagonal preconditioning, Engineering Analysis with Boundary Elements 27 (7) (2003) 727-750.

[39] S. Schneider, S. Marburg, Performance of iterative solvers for acoustic problems. part ii. acceleration by ilu-type preconditioner, Engineering Analysis with Boundary Elements 27 (7) (2003) 751-757.

995 [40] D. Ryckelynck, L. Hermanns, F. Chinesta, E. Alarcón, An efficient 'a priori'model reduction for boundary element models, Engineering Analysis with Boundary Elements 29 (8) (2005) 796-801.

[41] T. Liang, J. Wang, J. Xiao, L. Wen, Coupled be-fe based vibroacoustic modal analysis and frequency sweep using a generalized resolvent sampling method, Computer Methods in Applied Mechanics and Engineering 345 (2019) 518-538.

[42] J. Xiao, S. Meng, C. Zhang, C. Zheng, Resolvent sampling based rayleighritz method for large-scale nonlinear eigenvalue problems, Computer Methods in Applied Mechanics and Engineering 310 (2016) 33-57.

[43] W. E. Arnoldi, The principle of minimized iterations in the solution of the matrix eigenvalue problem, Quarterly of applied mathematics 9 (1) (1951) $17-29$.

[44] G. H. Golub, C. F. Van Loan, Matrix computations, Vol. 3, JHU press, 2012. 
[45] M. L. Parks, E. De Sturler, G. Mackey, D. D. Johnson, S. Maiti, Recycling krylov subspaces for sequences of linear systems, SIAM Journal on Scientific Computing 28 (5) (2006) 1651-1674.

[46] S. Keuchel, J. Biermann, O. von Estorff, A combination of the fast multipole boundary element method and krylov subspace recycling solvers, Engineering Analysis with Boundary Elements 65 (2016) 136-146.

[47] K. Carlberg, C. Farhat, An adaptive pod-krylov reduced-order model for structural optimization, in: 8th World Congress on Structural and Multidisciplinary Optimization, Lisbon, Portugal, Vol. 1, 2009, pp. 1-11.

[48] K. Carlberg, V. Forstall, R. Tuminaro, Krylov-subspace recycling via the pod-augmented conjugate-gradient method, SIAM Journal on Matrix Analysis and Applications 37 (3) (2016) 1304-1336.

[49] D. Ryckelynck, A priori hyperreduction method: an adaptive approach, Journal of Computational Physics 202 (1) (2005) 346 - 366. doi:https://doi.org/10.1016/j.jcp.2004.07.015.

25 URL http://www.sciencedirect.com/science/article/pii/ S002199910400289X

[50] S. Li, J. Trevelyan, W. Zhang, A Model Reduction Method for Boundary Element Method, Proceedings of the 24th UK Conference of the Association for Computational Mechanics in Engineering (2016).

[51] S. Li, J. Trevelyan, Z. Wu, H. Lian, D. Wang, W. Zhang, An adaptive svd-krylov reduced order model for surrogate based structural shape optimization through isogeometric boundary element method, Computer Methods in Applied Mechanics and Engineering 349 (2019) 312-338.

[52] D. Binion, X. Chen, A krylov enhanced proper orthogonal decomposition method for efficient nonlinear model reduction, Finite elements in analysis and design 47 (7) (2011) 728-738. 
[53] X. Xie, H. Zheng, S. Jonckheere, A. van de Walle, B. Pluymers, W. Desmet, Adaptive model reduction technique for large-scale dynamical systems with frequency-dependent damping, Computer Methods in Applied Mechanics and Engineering 332 (2018) 363-381.

[54] A. van de Walle, The power of model order reduction in vibroacoustics and its applications in model-based sensing, Ph.D. thesis, KU Leuven (2018).

[55] A. Burton, G. Miller, The application of integral equation methods to the numerical solution of some exterior boundary-value problems, Proceedings of the Royal Society of London. A. Mathematical and Physical Sciences 323 (1553) (1971) 201-210.

[56] H. A. Schenck, Improved integral formulation for acoustic radiation problems, The journal of the acoustical society of America 44 (1) (1968) 41-58.

[57] S. M. Kirkup, The boundary element method in acoustics, Integrated sound software, 2007.

[58] J. C. Mason, D. C. Handscomb, Chebyshev polynomials, Chapman and Hall/CRC, 2002.

[59] R. G. Bartle, D. R. Sherbert, Introduction to real analysis, Hoboken, NJ: Wiley, 2011.

[60] R. D. Ciskowski, C. A. Brebbia, Boundary element methods in acoustics, Springer, 1991.

[61] Å. Björck, Numerics of gram-schmidt orthogonalization, Linear Algebra and Its Applications 197 (1994) 297-316.

[62] V. C. Henriquez, P. M. Juhl, Openbem-an open source boundary element method software in acoustics, in: Internoise 2010, Vol. 7, Sociedade Portuguesa de Acustica (SPA) Lisbon, Portugal, 2010, pp. 5796-5805. 
[63] C. Geuzaine, J.-F. Remacle, Gmsh: A 3-d finite element mesh generator with built-in pre-and post-processing facilities, International journal for numerical methods in engineering 79 (11) (2009) 1309-1331. 